\title{
Investigation of subcombination internal resonances in cantilever beams
}

\author{
Haider N. Arafat and Ali H. Nayfeh * \\ Department of Engineering Science and Mechanics, \\ MC 0219, Virginia Polytechnic Institute and State \\ University, Blacksburg, VA 24061, USA
}

Received 29 May 1998

Revised 18 September 1998

Activation of subcombination internal resonances in transversely excited cantilever beams is investigated. The effect of geometric and inertia nonlinearities, which are cubic in the governing equation of motion, is considered. The method of time-averaged Lagrangian and virtual work is used to determine six nonlinear ordinary-differential equations governing the amplitudes and phases of the three interacting modes. Frequency- and force-response curves are generated for the case $\Omega \approx \omega_{4} \approx \frac{1}{2}\left(\omega_{2}+\omega_{5}\right)$. There are two possible responses: single-mode and three-mode responses. The singlemode periodic response is found to undergo supercritical and subcritical pitchfork bifurcations, which result in threemode interactions. In the case of three-mode responses, there are conditions where the low-frequency mode dominates the response, resulting in high-amplitude quasiperiodic oscillations.

Keywords: beams, subcombination internal resonance, bifurcations

\section{Introduction}

Sometimes exciting a structure near one of its natural frequencies can result in a complex response consisting of two or more modes. This coupling among the modes is usually attributed to internal resonances inherent in a nonlinear system. One type consists of combination and subcombination internal resonances. For systems with quadratic nonlinearities, combination internal resonances have the form $\omega_{k} \approx \omega_{i} \pm \omega_{j}$, where the $k$ th mode is the directly excited mode. For systems with cubic nonlinearities, combination internal reso-

\footnotetext{
${ }^{*}$ Corresponding author. Tel.: +1 540231 5453; Fax: +1 540231 2290; E-mail: anayfeh@vt.edu.
}

nances have the forms $\omega_{m} \approx \omega_{k} \pm \omega_{i} \pm \omega_{j}$ and $\omega_{m} \approx$ $2 \omega_{i} \pm \omega_{j}$ and subcombination internal resonances have the form $\omega_{m} \approx \frac{1}{2}\left(\omega_{i} \pm \omega_{j}\right)$, where the $m$ th mode is the directly excited mode (Nayfeh and Mook [7,8]).

Tezak et al. [14], Yamamoto et al. [16,17], and Ibrahim et al. [4] investigated combination internal resonances in extensional beams, whereas Zaretzky and Crespo da Silva [18] investigated these resonances in inextensional beams. Sridhar et al. [11,12] and Hadian and Nayfeh [3] theoretically investigated the combination internal resonance $\omega_{3} \approx 2 \omega_{2}+\omega_{1}$ in clamped circular plates. They found that multimode vibrations occur only when $\Omega \approx \omega_{3}$. Sridhar et al. [11,12] and Hadian and Nayfeh [3] found that the equilibrium solutions of the modulation equations undergo a Hopf bifurcation, which results in periodic, quasiperiodic, and chaotic motions. In these systems, the nonlinearity is cubic. On the other hand, Bux and Roberts [1], Cartmell and Roberts [2], and Nayfeh et al. [9] investigated combination internal resonances in systems with quadratic nonlinearities, namely two-beam structures.

Two recent experiments that clearly show the activation of subcombination internal resonances in structures were conducted by $\mathrm{Oh}$ and Nayfeh [10] and Tabaddor and Nayfeh [13]. Oh and Nayfeh [10] investigated the response of $(-75 / 75 / 75 /-75 / 75 /-75)_{s}$ epoxy-graphite cantilever plates that are transversely excited at $\Omega \approx \omega_{8} \approx \frac{1}{2}\left(\omega_{2}+\omega_{13}\right)$ where $\omega_{2}=$ $132.52 \mathrm{~Hz}, \omega_{8}=1036.90 \mathrm{~Hz}$, and $\omega_{13}=1963.90 \mathrm{~Hz}$. They found that, as the forcing amplitude increases, the single-mode response undergoes a supercritical pitchfork bifurcation and the response becomes quasiperiodic. Further, as the forcing amplitude increases, the response becomes dominated by the low-frequency mode.

In the second experiment, Tabaddor and Nayfeh [13] investigated the nonlinear response of a cantilever metallic beam to a transverse harmonic excitation. When the beam was mounted vertically, they found that direct excitation of the fourth mode results in the activation of the second and fifth modes via a subcombination internal resonance. When the beam was 
mounted horizontally, they observed two subcombination internal resonances: $\omega_{4} \approx \frac{1}{2}\left(\omega_{2}+\omega_{5}\right)$ (same case as when mounted vertically) and $\omega_{6} \approx \frac{1}{2}\left(\omega_{2}+\omega_{8}\right)$. In this paper, we investigate subcombination internal resonances in beams.

Usually, one assumes that the response amplitude of a structure to a low-amplitude high-frequency excitation is quite small and therefore the vibrations are safe. However, in certain cases, modal interactions can result in transferring energy to a low-frequency mode and in the process produce a large motion. Unless one plans for such a situation in a design, failure remains a possibility. The experiments of Oh and Nayfeh [10] and Tabaddor and Nayfeh [13] clearly show that a subcombination internal resonance can be a mechanism for this type of behavior, and hence it is very important to gain a theoretical understanding of it.

In this paper, we investigate the response of a uniform homogeneous cantilever beam to a primary resonance of one of its modes, which is involved in a subcombination internal resonance with two other modes. Both geometric and inertia nonlinearities are included, but the effects of shear deformation and rotatory inertia are neglected as the beam considered is assumed to be relatively long and thin (Timoshenko [15]). We analyze the response using the method of time-averaged Lagrangian and virtual work (Nayfeh [5,6]). Then, we use the modulation equations governing the amplitudes and phases to determine the behavior of the equilibrium solutions.

\section{Problem Formulation}

For planar flexural oscillations, the nondimensional equation of motion is given by

$$
\begin{aligned}
\ddot{v}+ & c \dot{v}+v^{i v}=-\left\{\left(v^{\prime 2} v^{\prime \prime \prime}+v^{\prime} v^{\prime \prime 2}\right)\right. \\
& \left.+\frac{1}{2}\left(v^{\prime} \frac{\partial^{2}}{\partial t^{2}} \int_{1}^{s} \int_{0}^{s} v^{\prime 2} \mathrm{~d} s \mathrm{~d} s\right)\right\}^{\prime}+F \cos (\Omega t)
\end{aligned}
$$

and the boundary conditions are

$$
\begin{array}{rlll}
v=0 & \text { and } & v^{\prime}=0 \quad \text { at } s=0, \\
v^{\prime \prime}=0 & \text { and } \quad v^{\prime \prime \prime}=0 \quad \text { at } s=1,
\end{array}
$$

where the prime denotes differentiation with respect to the axial coordinate $s$ and the overdot denotes differentiation with respect to time $t$. Distances are nondi- mensionalized using the undeformed length $L$ of the beam and time is nondimensionalized using the characteristic time $\sqrt{m L^{4} / E I}$. The corresponding nondimensional Lagrangian and virtual work are given by

$$
\begin{aligned}
\mathcal{L}= & \int_{0}^{1}\left\{\frac{1}{2} \dot{v}^{2}+\frac{1}{2}\left(\frac{1}{2} \frac{\partial}{\partial t} \int_{0}^{s} v^{\prime 2} \mathrm{~d} s\right)^{2}\right. \\
& \left.-\frac{1}{2}\left(v^{\prime \prime 2}+v^{\prime 2} v^{\prime \prime 2}\right)\right\} \mathrm{d} s, \\
\delta W= & \int_{0}^{1} Q_{v} \delta v \mathrm{~d} s \\
= & \int_{0}^{1}\{F \cos (\Omega t)-c \dot{v}\} \delta v \mathrm{~d} s .
\end{aligned}
$$

Next, we consider the subcombination internal resonance $\omega_{m} \approx \frac{1}{2}\left(\omega_{l}+\omega_{n}\right)$ when the $m$ th mode is excited with a primary resonance (i.e., $\Omega \approx \omega_{m}$ ). In the presence of damping, the steady-state response of the beam will consist only of the $l$ th, $m$ th, and $n$th modes involved in the internal resonance. Therefore, we approximate $v(s, t)$ as

$$
\begin{aligned}
v(s, t) & \approx v_{l}(s, t)+v_{m}(s, t)+v_{n}(s, t) \\
& \approx \phi_{l}(s) \eta_{l}(t)+\phi_{m}(s) \eta_{m}(t)+\phi_{n}(s) \eta_{n}(t),
\end{aligned}
$$

where the $\phi_{j}(s)$ are the orthonormal mode shapes. For cantilever beams,

$$
\begin{aligned}
& \phi_{j}(s)=\cosh \left(z_{j} s\right)-\cos \left(z_{j} s\right) \\
& \quad+\frac{\cos \left(z_{j}\right)+\cosh \left(z_{j}\right)}{\sin \left(z_{j}\right)+\sinh \left(z_{j}\right)}\left[\sin \left(z_{j} s\right)-\sinh \left(z_{j} s\right)\right],
\end{aligned}
$$

where $z_{j}$ is the $j$ th root of $1+\cos (z) \cosh (z)=0$. The nondimensional natural frequencies are given by

$$
\omega_{j}=z_{j}^{2}
$$

The first five natural frequencies are $\omega_{1}=3.516, \omega_{2}=$ 22.0345, $\omega_{3}=61.6972, \omega_{4}=120.9019$, and $\omega_{5}=$ 199.8595 .

A first-order uniform expansion for the $\eta_{j}$ is taken in the form

$\eta_{j}=\varepsilon\left[A_{j}\left(T_{2}\right) \mathrm{e}^{\mathrm{i} \omega_{j} T_{0}}+\bar{A}_{j}\left(T_{2}\right) \mathrm{e}^{-\mathrm{i} \omega_{j} T_{0}}\right]+\cdots$,

where the overbar denotes the complex conjugate, $\varepsilon$ is a small nondimensional bookkeeping parameter, $T_{0}=t$, and $T_{2}=\varepsilon^{2} t$ (Nayfeh [5]). In order that the nonlin- 
earity, damping, and resonance balance, we scale $F$ as $\varepsilon^{3} F$ and $c$ as $\varepsilon^{2} c$. Also, we introduce the detuning parameters $\sigma_{1}$ and $\sigma_{2}$ such that

$$
\begin{aligned}
\omega_{m} & =\frac{1}{2}\left(\omega_{l}+\omega_{n}\right)+\varepsilon^{2} \sigma_{1} \quad \text { and } \\
\Omega & =\omega_{m}+\varepsilon^{2} \sigma_{2} .
\end{aligned}
$$

We substitute Eqs (6), (9), and (10) into Eqs (4) and (5), perform the spatial integrations, retain the slowly varying terms, and obtain the time-averaged Lagrangian and virtual work as

$$
\begin{aligned}
\frac{\langle\mathcal{L}\rangle}{\varepsilon^{4}}= & \mathrm{i} \omega_{l}\left(A_{l} \bar{A}_{l}^{\prime}-\bar{A}_{l} A_{l}^{\prime}\right) \\
& +\mathrm{i} \omega_{m}\left(A_{m} \bar{A}_{m}^{\prime}-\bar{A}_{m} A_{m}^{\prime}\right) \\
& +\mathrm{i} \omega_{n}\left(A_{n} \bar{A}_{n}^{\prime}-\bar{A}_{n} A_{n}^{\prime}\right) \\
& -S_{l m} A_{l} \bar{A}_{l} A_{m} \bar{A}_{m}-S_{l n} A_{l} \bar{A}_{l} A_{n} \bar{A}_{n} \\
& -S_{m n} A_{m} \bar{A}_{m} A_{n} \bar{A}_{n}-\frac{1}{2} S_{l l} A_{l}^{2} \bar{A}_{l}^{2} \\
& -\frac{1}{2} S_{m m} A_{m}^{2} \bar{A}_{m}^{2}-\frac{1}{2} S_{n n} A_{n}^{2} \bar{A}_{n}^{2} \\
& -\Lambda\left(A_{m}^{2} \bar{A}_{l} \bar{A}_{n} \mathrm{e}^{\mathrm{i} \sigma_{1} T_{2}}\right. \\
& \left.+\bar{A}_{m}^{2} A_{l} A_{n} \mathrm{e}^{-2 \mathrm{i} \sigma_{1} T_{2}}\right)+\cdots, \\
\frac{\langle\delta W\rangle}{\varepsilon^{4}}= & Q_{l} \delta A_{l}+Q_{m} \delta A_{m} \\
& +Q_{n} \delta A_{n}+\mathrm{cc}+\cdots,
\end{aligned}
$$

where

$$
\begin{aligned}
S_{j j}= & 2 \int_{0}^{1}\left(3 \phi_{j}^{\prime 2} \phi_{j}^{\prime \prime 2}-\omega_{j}^{2} \gamma_{j j}^{2}\right) \mathrm{d} s, \\
S_{j k}= & \int_{0}^{1}\left[2 \phi_{j}^{\prime 2} \phi_{k}^{\prime \prime 2}+8 \phi_{j}^{\prime} \phi_{k}^{\prime} \phi_{j}^{\prime \prime} \phi_{k}^{\prime \prime}\right. \\
& \left.+2 \phi_{k}^{\prime 2} \phi_{j}^{\prime \prime 2}-2\left(\omega_{j}^{2}+\omega_{k}^{2}\right) \gamma_{j k}^{2}\right] \mathrm{d} s, \\
\Lambda= & \int_{0}^{1}\left[\phi_{l}^{\prime} \phi_{n}^{\prime} \phi_{m}^{\prime \prime 2}+2 \phi_{l}^{\prime} \phi_{m}^{\prime} \phi_{m}^{\prime \prime} \phi_{n}^{\prime \prime}\right. \\
& +2 \phi_{m}^{\prime} \phi_{n}^{\prime} \phi_{l}^{\prime \prime} \phi_{m}^{\prime \prime}+\phi_{m}^{\prime 2} \phi_{l}^{\prime \prime} \phi_{n}^{\prime \prime} \\
& -\left(\omega_{l} \omega_{m}+\omega_{m} \omega_{n}\right. \\
& \left.\left.-\omega_{m}^{2}-\omega_{l} \omega_{n}\right) \gamma_{l m} \gamma_{l n}\right] \mathrm{d} s, \\
\gamma_{j k}= & \int_{0}^{s} \phi_{j}^{\prime} \phi_{k}^{\prime} \mathrm{d} s,
\end{aligned}
$$

$$
\begin{aligned}
Q_{j} & =2 \mathrm{i} \omega_{j} \mu_{j} \bar{A}_{j}+\frac{1}{2} f \delta_{j m} \mathrm{e}^{-2 \mathrm{i} \sigma_{2} T_{2}}, \\
\mu_{j} & =\frac{1}{2} \int_{0}^{1} c \phi_{j}^{2} \mathrm{~d} s, \quad f=\int_{0}^{1} F \phi_{m} \mathrm{~d} s .
\end{aligned}
$$

Next, we apply Hamilton's extended principle

$$
\frac{\mathrm{d}}{\mathrm{d} T_{2}}\left(\frac{\partial\langle\mathcal{L}\rangle}{\partial \bar{A}_{j}^{\prime}}\right)-\frac{\partial\langle\mathcal{L}\rangle}{\partial \bar{A}_{j}}=\bar{Q}_{j}
$$

to Eqs (11) and (12) and obtain the following three complex-valued modulation equations:

$$
\begin{aligned}
-2 \mathrm{i} \omega_{l} & \left(A_{l}^{\prime}+\mu_{l} A_{l}\right) \\
= & S_{l l} A_{l}^{2} \bar{A}_{l}+S_{l m} A_{l} A_{m} \bar{A}_{m}+S_{l n} A_{l} A_{n} \bar{A}_{n} \\
& +\Lambda A_{m}^{2} \bar{A}_{n} \mathrm{e}^{2 \mathrm{i} \sigma_{1} T_{2}} \\
-2 \mathrm{i} \omega_{m}\left(A_{m}^{\prime}\right. & \left.+\mu_{m} A_{m}\right) \\
= & S_{m m} A_{m}^{2} \bar{A}_{m}+S_{l m} A_{m} A_{l} \bar{A}_{l}+S_{m n} A_{m} A_{n} \bar{A}_{n} \\
& +2 \Lambda \bar{A}_{m} A_{l} A_{n} \mathrm{e}^{-2 \mathrm{i} \sigma_{1} T_{2}}+\frac{1}{2} f \mathrm{e}^{\mathrm{i} \sigma_{2} T_{2}}, \\
-2 \mathrm{i} \omega_{n}\left(A_{n}^{\prime}+\mu_{n} A_{n}\right) & \\
= & S_{n n} A_{n}^{2} \bar{A}_{n}+S_{m n} A_{n} A_{m} \bar{A}_{m}+S_{l n} A_{n} A_{l} \bar{A}_{l} \\
& +\Lambda A_{m}^{2} \bar{A}_{l} \mathrm{e}^{2 \mathrm{i} \sigma_{1} T_{2}} .
\end{aligned}
$$

Equations (19)-(21) can be converted into polar form by introducing the transformation

$$
A_{j}=\frac{1}{2} a_{j} \mathrm{e}^{\mathrm{i} \beta_{j}}
$$

and separating the real and imaginary parts. The result is

$$
\begin{aligned}
a_{l}^{\prime}= & -\mu_{l} a_{l}-\frac{\Lambda}{8 \omega_{l}} a_{m}^{2} a_{n} \sin \gamma_{1}, \\
a_{m}^{\prime}= & -\mu_{m} a_{m}+\frac{\Lambda}{4 \omega_{m}} a_{l} a_{m} a_{n} \sin \gamma_{1} \\
& +\frac{f}{2 \omega_{m}} \sin \gamma_{2}, \\
a_{n}^{\prime}= & -\mu_{n} a_{n}-\frac{\Lambda}{8 \omega_{n}} a_{l} a_{m}^{2} \sin \gamma_{1}, \\
a_{l} \beta_{l}^{\prime}= & \frac{S_{l l}}{8 \omega_{l}} a_{l}^{3}+\frac{S_{l m}}{8 \omega_{l}} a_{l} a_{m}^{2} \\
& +\frac{S_{l n}}{8 \omega_{l}} a_{l} a_{n}^{2}+\frac{\Lambda}{8 \omega_{l}} a_{m}^{2} a_{n} \cos \gamma_{1},
\end{aligned}
$$




$$
\begin{aligned}
a_{m} \beta_{m}^{\prime}= & \frac{S_{m m}}{8 \omega_{m}} a_{m}^{3}+\frac{S_{l m}}{8 \omega_{m}} a_{l}^{2} a_{m} \\
& +\frac{S_{m n}}{8 \omega_{m}} a_{m} a_{n}^{2}+\frac{\Lambda}{4 \omega_{m}} a_{l} a_{m} a_{n} \cos \gamma_{1} \\
& -\frac{f}{2 \omega_{m}} \cos \gamma_{2}, \\
a_{n} \beta_{n}^{\prime}= & \frac{S_{n n}}{8 \omega_{n}} a_{n}^{3}+\frac{S_{l n}}{8 \omega_{n}} a_{l}^{2} a_{n} \\
& +\frac{S_{m n}}{8 \omega_{n}} a_{m}^{2} a_{n}+\frac{\Lambda}{8 \omega_{n}} a_{l} a_{m}^{2} \cos \gamma_{1},
\end{aligned}
$$

where

$$
\begin{aligned}
& \gamma_{1}=2 \sigma_{1} T_{2}+2 \beta_{m}-\beta_{l}-\beta_{n} \quad \text { and } \\
& \gamma_{2}=\sigma_{2} T_{2}-\beta_{m} .
\end{aligned}
$$

There are two possible solutions: $a_{l}=0, a_{n}=0$, and $a_{m} \neq 0$ and $a_{l} \neq 0, a_{n} \neq 0$, and $a_{m} \neq 0$. When $a_{l}$, $a_{m}$, and $a_{n} \neq 0$, Eqs (26)-(29) can be combined into the following two first-order differential equations for $\gamma_{1}$ and $\gamma_{2}$ :

$$
\begin{aligned}
\gamma_{1}^{\prime}= & 2 \sigma_{1}+\frac{1}{4}\left(\frac{S_{l m}}{\omega_{m}}-\frac{S_{l l}}{2 \omega_{l}}-\frac{S_{l n}}{2 \omega_{n}}\right) a_{l}^{2} \\
& +\frac{1}{4}\left(\frac{S_{m m}}{\omega_{m}}-\frac{S_{l m}}{2 \omega_{l}}-\frac{S_{m n}}{2 \omega_{n}}\right) a_{m}^{2} \\
& +\frac{1}{4}\left(\frac{S_{m n}}{\omega_{m}}-\frac{S_{l n}}{2 \omega_{l}}-\frac{S_{n n}}{2 \omega_{n}}\right) a_{n}^{2} \\
& +\frac{\Lambda}{2}\left(\frac{a_{l} a_{n}}{\omega_{m}}-\frac{a_{m}^{2} a_{n}}{4 \omega_{l} a_{l}}-\frac{a_{m}^{2} a_{l}}{4 \omega_{n} a_{n}}\right) \cos \gamma_{1} \\
& -\frac{f}{\omega_{m} a_{m}} \cos \gamma_{2}, \\
\gamma_{2}^{\prime}= & \sigma_{2}-\frac{S_{l m}}{8 \omega_{m}} a_{l}^{2}-\frac{S_{m m}}{8 \omega_{m}} a_{m}^{2}-\frac{S_{m n}}{8 \omega_{m}} a_{n}^{2} \\
& -\frac{\Lambda}{4 \omega_{m}} a_{l} a_{n} \cos \gamma_{1}+\frac{f}{2 \omega_{m} a_{m}} \cos \gamma_{2} .
\end{aligned}
$$

Equations (23)-(25), (30), and (31) constitute an autonomous system of five first-order ordinary-differential equations governing the behavior of the amplitudes $a_{l}, a_{m}$, and $a_{n}$ and the phases $\gamma_{1}$ and $\gamma_{2}$.

The equilibrium solutions (fixed points) of Eqs (23)(29) correspond to setting $a_{l}^{\prime}, a_{m}^{\prime}, a_{n}^{\prime}, \gamma_{1}^{\prime}$, and $\gamma_{2}^{\prime}=0$. There are two possibilities: (a) $a_{l}$ and $a_{n}=0$ while $a_{m} \neq 0$ and (b) $a_{l}, a_{m}$, and $a_{n} \neq 0$. In the first case, Eqs (24), (27), and (29b) reduce to

$$
\begin{aligned}
& \mu_{m} a_{m}=\frac{f}{2 \omega_{m}} \sin \gamma_{2}, \\
& a_{m}\left(\frac{S_{m m}}{8 \omega_{m}} a_{m}^{2}-\sigma_{2}\right)=\frac{f}{2 \omega_{m}} \cos \gamma_{2}
\end{aligned}
$$

Squaring and adding Eqs (32) and (33), we obtain a single equation for $a_{m}$ in terms of the forcing amplitude $F$ and the detuning parameter $\sigma_{2}$. The result is

$$
a_{m}^{2}\left[\left(\frac{S_{m m}}{8 \omega_{m}} a_{m}^{2}-\sigma_{2}\right)^{2}+\mu_{m}^{2}\right]=\frac{f^{2}}{4 \omega_{m}^{2}} .
$$

To determine the stability of this solution, we consider the linearized complex-valued modulation equations. We substitute

$$
\begin{aligned}
& A_{l}=C_{l} \mathrm{e}^{\lambda T_{2}+2 \mathrm{i}\left(\sigma_{1} T_{2}+\beta_{m}\right)}, \\
& A_{n}=C_{n} \mathrm{e}^{\bar{\lambda} T_{2}},
\end{aligned}
$$

where $C_{l}$ and $C_{n}$ are constants, into Eqs (19) and (21), use Eq. (29b), and obtain

$$
\begin{aligned}
& {\left[-2 \omega_{l}\left(\lambda+\mu_{l}\right)+4 \omega_{l}\left(\sigma_{1}+\sigma_{2}\right)-\frac{S_{l m}}{4} a_{m}^{2}\right] C_{l}} \\
& -\frac{\Lambda}{4} a_{m}^{2} \bar{C}_{n}=0, \\
& -\frac{\Lambda}{4} a_{m}^{2} \bar{C}_{l}+\left[-2 \mathrm{i} \omega_{n}\left(\bar{\lambda}+\mu_{n}\right)-\frac{S_{m n}}{4} a_{m}^{2}\right] C_{n}=0 .
\end{aligned}
$$

The characteristic equation for $\lambda$ is given by

$$
\begin{aligned}
\lambda^{2}+ & {\left[\left(\mu_{l}+\mu_{n}\right)+2 \mathrm{i}\left(\sigma_{1}+\sigma_{2}\right)\right.} \\
& \left.+\frac{\mathrm{i}}{8}\left(\frac{S_{m n}}{\omega_{n}}-\frac{S_{l m}}{\omega_{l}}\right) a_{m}^{2}\right] \lambda \\
& +\left\{\mu_{l} \mu_{n}+2 \mathrm{i} \mu_{n}\left(\sigma_{1}+\sigma_{2}\right)\right. \\
& +\left[\frac{\mathrm{i}}{8}\left(\frac{S_{m n}}{\omega_{n}} \mu_{l}-\frac{S_{l m}}{\omega_{l}} \mu_{n}\right)\right. \\
& \left.-\frac{1}{4}\left(\sigma_{1}+\sigma_{2}\right) \frac{S_{m n}}{\omega_{n}}\right] a_{m}^{2} \\
& \left.+\left(\frac{S_{l m} S_{m n}-\Lambda^{2}}{64 \omega_{l} \omega_{n}}\right) a_{m}^{4}\right\}=0 .
\end{aligned}
$$

Equations (35) and (36) show that $A_{l}$ and $A_{n}$ grow exponentially with time and hence the single-mode solu- 


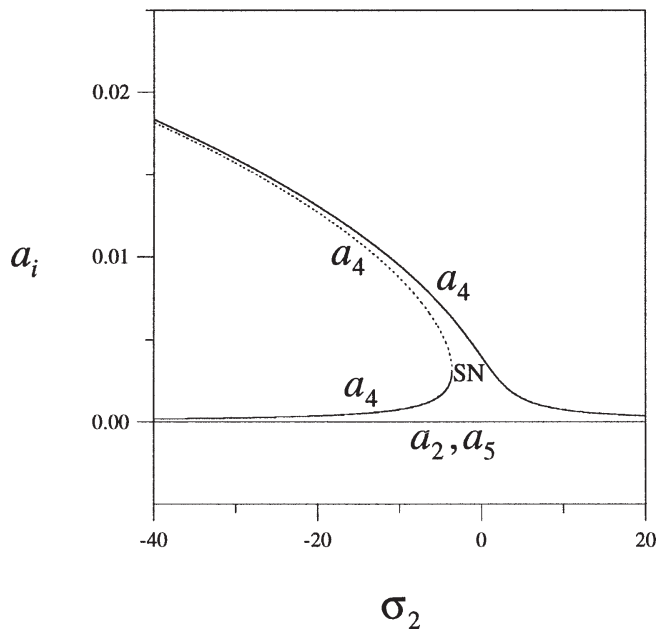

Fig. 1. Frequency-response curves for the case $\Omega \approx \omega_{4}$ $\approx \frac{1}{2}\left(\omega_{2}+\omega_{5}\right)$ when $F=10, \sigma_{1}=-5, \mu_{2}=0.0635, \mu_{4}=0.0573$, and $\mu_{5}=0.0400$. Solid lines $(-)$ denote stable fixed points and dotted lines $(\cdots)$ denote unstable fixed points. SN denotes a saddle-node bifurcation.

tion loses stability when the real part of $\lambda$ crosses the imaginary axis from the left-half to the right-half of the complex plane.

For the second case, a closed-form solution is not available so that numerical methods are employed. It follows from Eqs (23) and (25) that

$$
a_{l}^{2}=\frac{\mu_{n} \omega_{n}}{\mu_{l} \omega_{l}} a_{n}^{2}
$$

We note that the internal resonance defined in Eq. (10) cannot be activated if the excitation frequency is near either $\omega_{l}$ or $\omega_{n}$. This is so because in these cases Eq. (40) would be replaced with

$$
a_{n}^{2}=-\frac{\mu_{m} \omega_{m}}{\mu_{n} \omega_{n}} a_{m}^{2} \text { or } a_{l}^{2}=-\frac{\mu_{m} \omega_{m}}{\mu_{l} \omega_{l}} a_{m}^{2},
$$

which are only satisfied when $a_{m}=a_{n}=0$ or $a_{m}=$ $a_{l}=0$, respectively.

To compare our analysis with the experimental results of Tabaddor and Nayfeh [13], we next consider the resonance case $\Omega \approx \omega_{4} \approx \frac{1}{2}\left(\omega_{2}+\omega_{5}\right)$. In Table 1 , we present the corresponding numerical values for the constants $S_{j k}, \Lambda$, and $f$.

In Figs 1 and 2, we present typical frequencyresponse curves when the forcing amplitude $F=10$. In Fig. 1, $\sigma_{1}=-5$, and in Fig. 2, $\sigma_{1}=10$. We note that the curves for both the single- and three-mode solutions are bent to the left, indicating that the effect of the nonlinearities is softening. It follows from Fig. 1
Table 1

Values of the constants $S_{j k}, \Lambda$, and $f$ for $\Omega \approx \omega_{4} \approx \frac{1}{2}\left(\omega_{2}+\omega_{5}\right)$

\begin{tabular}{cr}
\hline$S_{l l}$ & -142282.2707 \\
$S_{m m}$ & $-1.1623 \times 10^{8}$ \\
$S_{n n}$ & $-8.9508 \times 10^{8}$ \\
$S_{l m}$ & -927411.9209 \\
$S_{l n}$ & $-1.4422 \times 10^{6}$ \\
$S_{m n}$ & $-5.7032 \times 10^{7}$ \\
$\Lambda$ & $5.8709 \times 10^{6}$ \\
$f$ & $0.1819 F$ \\
\hline
\end{tabular}

that only the fourth mode is excited; there are three branches: two are stable and one is unstable. Therefore, depending on the initial conditions, the response may have either a small or a large amplitude. On the other hand, when $\sigma_{1}=10$, it follows from Fig. 2 that the high-amplitude single-mode solution loses stability via two bifurcations: a supercritical pitchfork PF1 and a subcritical pitchfork PF2. From the first bifurcation point, branches of stable three-mode solutions emanate smoothly from the single-mode solution. Once the solution is multimodal, the amplitude of the second mode continues to grow as $\sigma_{2}$ is decreased, and eventually its amplitude becomes larger than that of the fourth mode. Consequently, the beam's oscillations may grow to be dangerously large, in qualitative agreement with the experimental results of Tabaddor and Nayfeh [13]. From the second bifurcation point, branches of unstable three-mode solutions emanate and a jump occurs. We note that, in the region between the two pitchfork bifurcation points, stable single-mode solutions coexist with the three-mode solutions and hence the beam response depends on the initial conditions.

Going back to Eqs (34) and (39), we note that although the single-mode response is independent of the detuning parameter $\sigma_{1}$, its stability is dependent on $\sigma_{1}$. This can be clearly seen by comparing Figs 1 and 2(a). Changing $\sigma_{1}$ does not affect the shape of the single-mode curves, but it does affect their stability. We should also note that, for a lightly damped system, both the low-amplitude and high-amplitude single-mode solutions lose stability, giving rise to three-mode interactions.

The steady-state response of the free end of the beam is shown in Fig. 3 when $\sigma_{1}=10$ and $F=10$. The values of $\sigma_{2}$ in parts (a) and (b) are -10.5 and -11.0 , respectively, which lie immediately above and below the supercritical pitchfork bifurcation PF1. As $\sigma_{2}$ is decreased past PF1, the constant amplitude unimodal response becomes modulated due to contributions from 
the second and fifth modes. As $\sigma_{2}$ decreases further, these contributions become more pronounced, causing the amplitude of the modulated response to increase

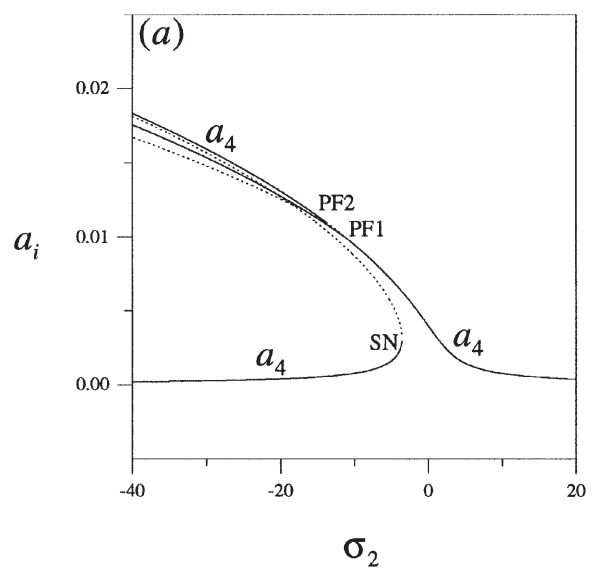

considerably, as shown in Fig. 3(c) for $\sigma_{2}=-20$.

In Figs 4 and 5 we present amplitudes-response curves when $\sigma_{1}=10$. In Fig. 4, $\sigma_{2}=-10$, and in

Fig. 2. Frequency-response curves for the case $\Omega \approx \omega_{4} \approx \frac{1}{2}\left(\omega_{2}+\omega_{5}\right)$ when $F=10, \sigma_{1}=10, \mu_{2}=0.0635, \mu_{4}=0.0573$, and $\mu_{5}=0.0400$. Solid lines (-) denote stable fixed points and dotted lines $(\cdots)$ denote unstable fixed points. PF1 and PF2 denote supercritical and subcritical pitchfork bifurcations, respectively, and SN denotes a saddle-node bifurcation.
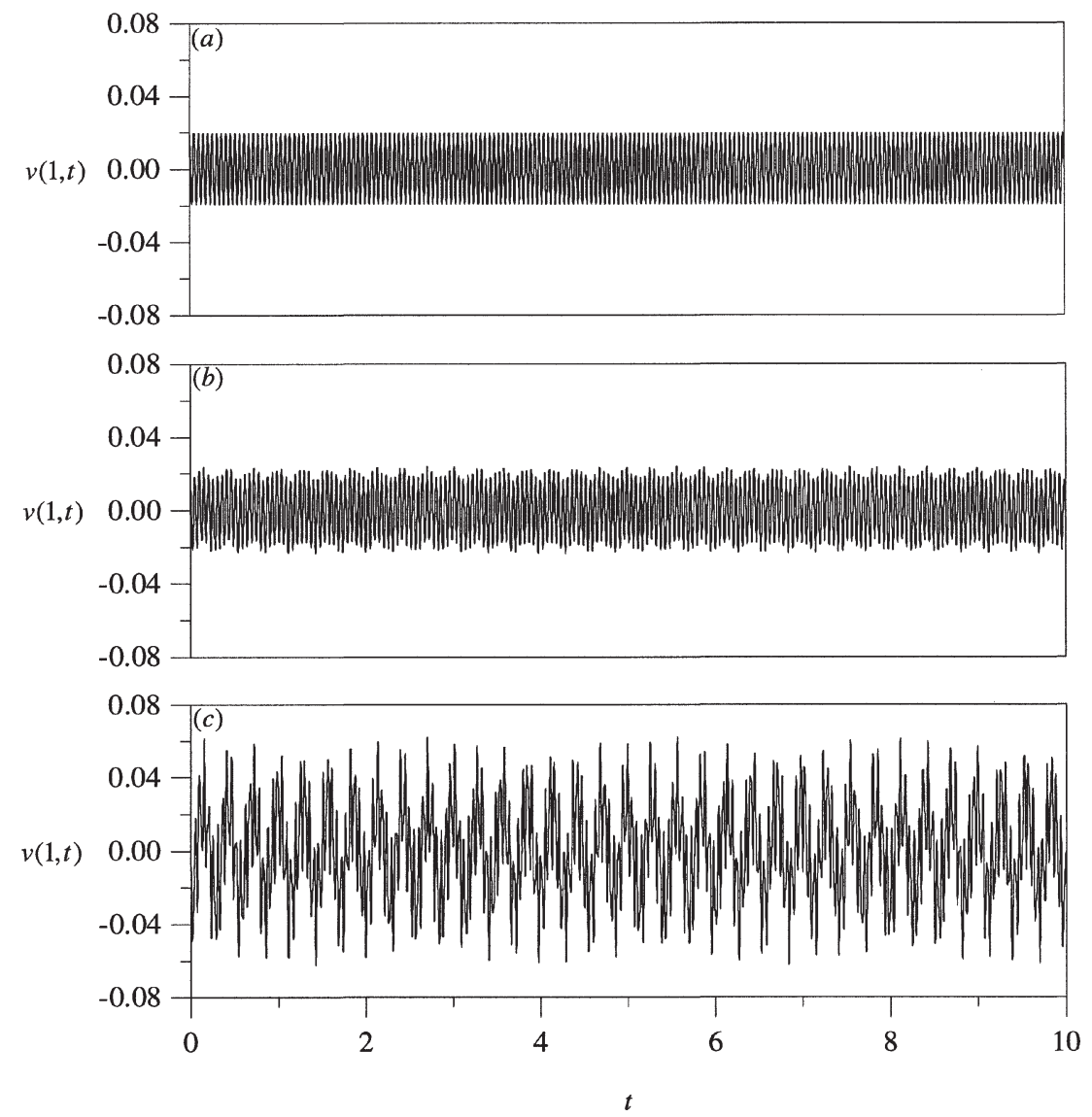

Fig. 3. Steady-state response of the beam at the free end $s=1$ when $\Omega \approx \omega_{4} \approx \frac{1}{2}\left(\omega_{2}+\omega_{5}\right), \sigma_{1}=10, F=10$, and $\varepsilon=0.1$. (a) $\sigma_{2}=-10.5$, (b) $\sigma_{2}=-11$, (c) $\sigma_{2}=-20$. 


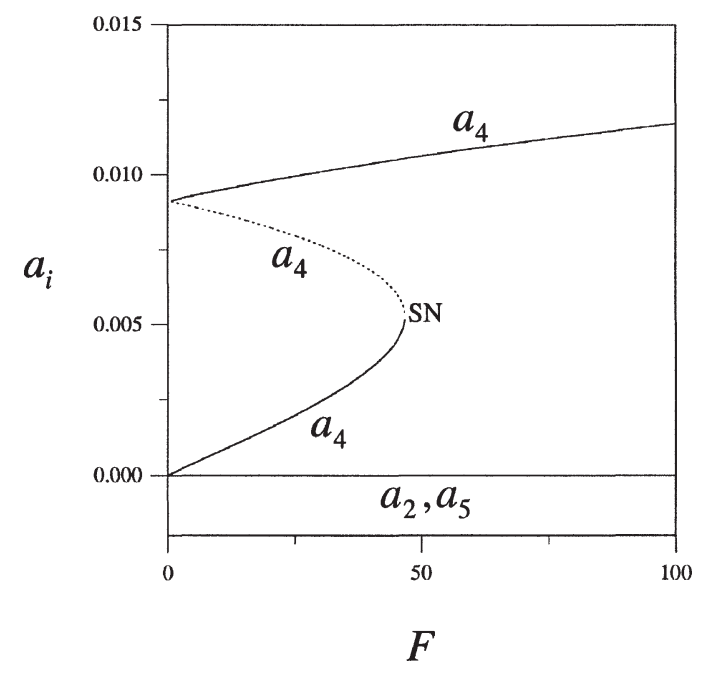

Fig. 4. Amplitude-response curves for the case $\Omega \approx \omega_{4} \approx \frac{1}{2}\left(\omega_{2}+\omega_{5}\right)$ when $\sigma_{1}=10, \sigma_{2}=-10, \mu_{2}=0.0635, \mu_{4}=0.0573$, and $\mu_{5}=0.0400$. Solid lines $(-)$ denote stable fixed points and dotted lines $(\cdots)$ denote unstable fixed points. SN denotes a saddle-node bifurcation.
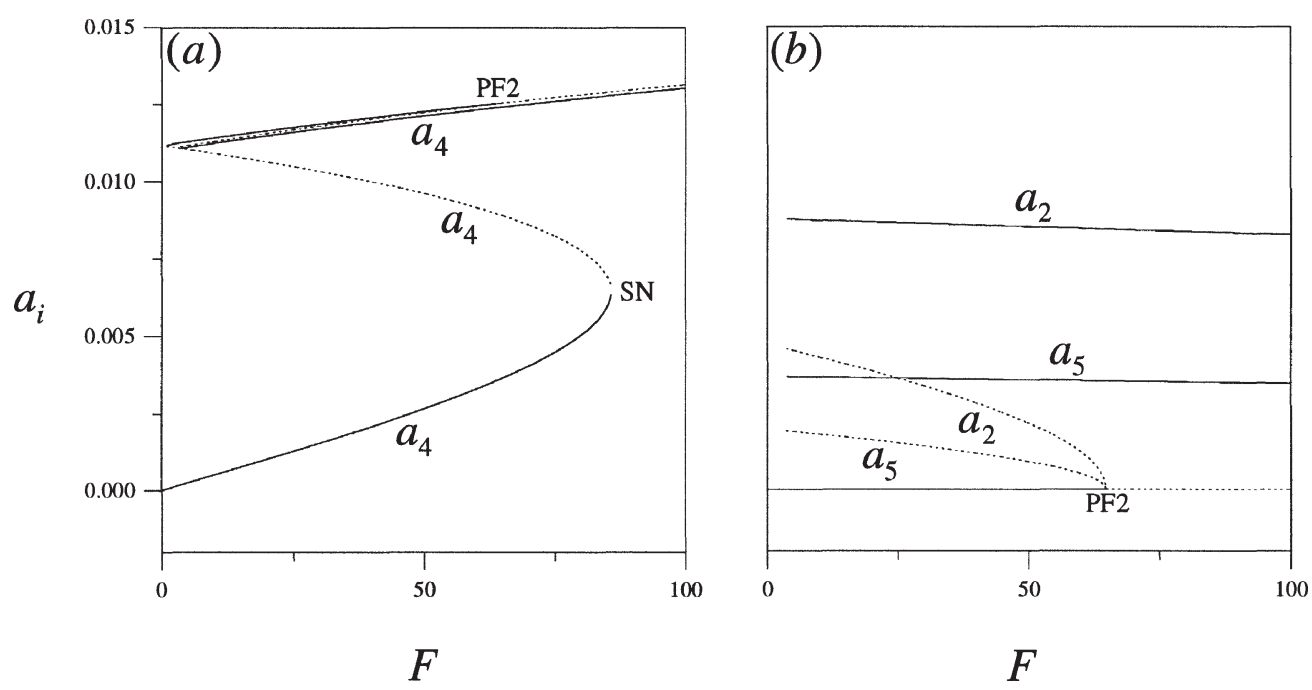

Fig. 5. Amplitude-response curves for the case $\Omega \approx \omega_{4} \approx \frac{1}{2}\left(\omega_{2}+\omega_{5}\right)$ when $\sigma_{1}=10, \sigma_{2}=-15, \mu_{2}=0.0635, \mu_{4}=0.0573$, and $\mu_{5}=0.0400$. Solid lines $(-)$ denote stable fixed points and dotted lines $(\cdots)$ denote unstable fixed points. PF2 denotes a subcritical pitchfork bifurcations and $\mathrm{SN}$ denotes a saddle-node bifurcation.

Fig. 5, $\sigma_{2}=-15$. It follows from Fig. 4 that only the fourth mode is excited. When $\sigma_{2}=-15$, the upper branch loses stability via a subcritical pitchfork bifurcation as $F$ is increased, resulting in a sudden jump that marks the activation of a three-mode response.

\section{Conclusion}

The planar response of cantilever beams to subcombination internal resonances has been investigated. We used the method of time-averaged Lagrangian and virtual work to derive a set of six first-order nonlinear ordinary-differential equations governing the modulation of the amplitudes and phases. We found that the single-mode response can lose stability via supercritical and subcritical pitchfork bifurcations, giving rise to three-mode responses. Depending on the forcing frequency and amplitude, contributions from the low-frequency mode may be significant, resulting in large-amplitude oscillations of the beam, in qualitative agreement with the experimental results of Tabaddor and Nayfeh [13]. 


\section{Acknowledgment}

This work was supported by the National Science Foundation under Grant No. CMS-9423774.

\section{References}

[1] S.L. Bux and J.W. Roberts, Non-linear vibratory interactions in systems of coupled beams, J. Sound and Vibration 104 (1986), 497-520.

[2] M.P. Cartmell and J.W. Roberts, Simultaneous combination resonances in an autoparametrically resonant system, J. Sound and Vibration 123 (1988), 81-101.

[3] J. Hadian and A.H. Nayfeh, Modal interactions in circular plates, J. Sound and Vibration 142 (1990), 279-292.

[4] R.A. Ibrahim, A. Afaneh and B. Lee, Non-linear modal interaction in a clamped-clamped beam: Part I - deterministic approach, in: ASME Vibration Analysis-Analytical and Computational Conference, DE-Vol. 37, 1991, pp. 97-107.

[5] A.H. Nayfeh, Introduction to Perturbation Techniques, WileyInterscience, New York, 1981.

[6] A.H. Nayfeh, On the discretization of weekly nonlinear spatially continuous systems, in: Differential Equations and Chaos Lectures on Selected Topics, N.H. Ibragimov, F.M. Mahomed, D.P. Mason and D. Sherwell, eds, New Age International, New Delhi, 1996, pp. 3-39.

[7] A.H. Nayfeh and D.T. Mook, A saturation phenomenon in the forced response of systems with quadratic nonlinearities, in: Proc. VIIIth Int. Conf. on Nonlinear Oscillations, Prague, Czech Republic, 1978, pp. 511-516.
[8] A.H. Nayfeh and D.T. Mook, Nonlinear Oscillations, WileyInterscience, New York, 1979.

[9] T.A. Nayfeh, A.H. Nayfeh and D.T. Mook, A theoretical and experimental investigation of a three-degree-of-freedom structure, Nonlinear Dynamics 6 (1994), 353-374.

[10] K. Oh and A.H. Nayfeh, Nonlinear combination resonances in cantilever composite plates, Nonlinear Dynamics 11 (1996), 143-169.

[11] S. Sridhar, D.T. Mook and A.H. Nayfeh, Non-linear resonances in the forced responses of plates, Part I: symmetric responses of circular plates, J. Sound and Vibration 41 (1975), 359-373.

[12] S. Sridhar, D.T. Mook and A.H. Nayfeh, Non-linear resonances in the forced responses of plates, Part II: asymmetric responses of circular plates, J. Sound and Vibration 59 (1978), 159-170.

[13] M. Tabaddor and A.H. Nayfeh, An experimental investigation of multimode responses in a cantilever beam, J. Vibration and Acoustics, Trans. ASME 119 (1997), 532-538.

[14] E.G. Tezak, D.T. Mook and A.H. Nayfeh, Nonlinear analysis of the lateral response of columns to periodic loads, J. Mechanical Design, Trans. ASME 100 (1978), 651-659.

[15] S. Timoshenko, Vibration Problems in Engineering, Van Nostrand, New York, 1928.

[16] T. Yamamoto, K. Yasuda and N. Tei, Summed and differential harmonic oscillations in a slender beam, Bulletin JSME 24 (1981), 1214-1222.

[17] T. Yamamoto, K. Yasuda and N. Tei, Super summed and differential harmonic oscillations in a slender beam, Bulletin JSME 25 (1982), 959-968.

[18] C.L. Zaretzky and M.R.M. Crespo da Silva, Nonlinear flexuralflexural-torsional interactions in beams including the effect of torsional dynamics. II: Combination resonance, Nonlinear Dynamics 5 (1994), 161-180. 

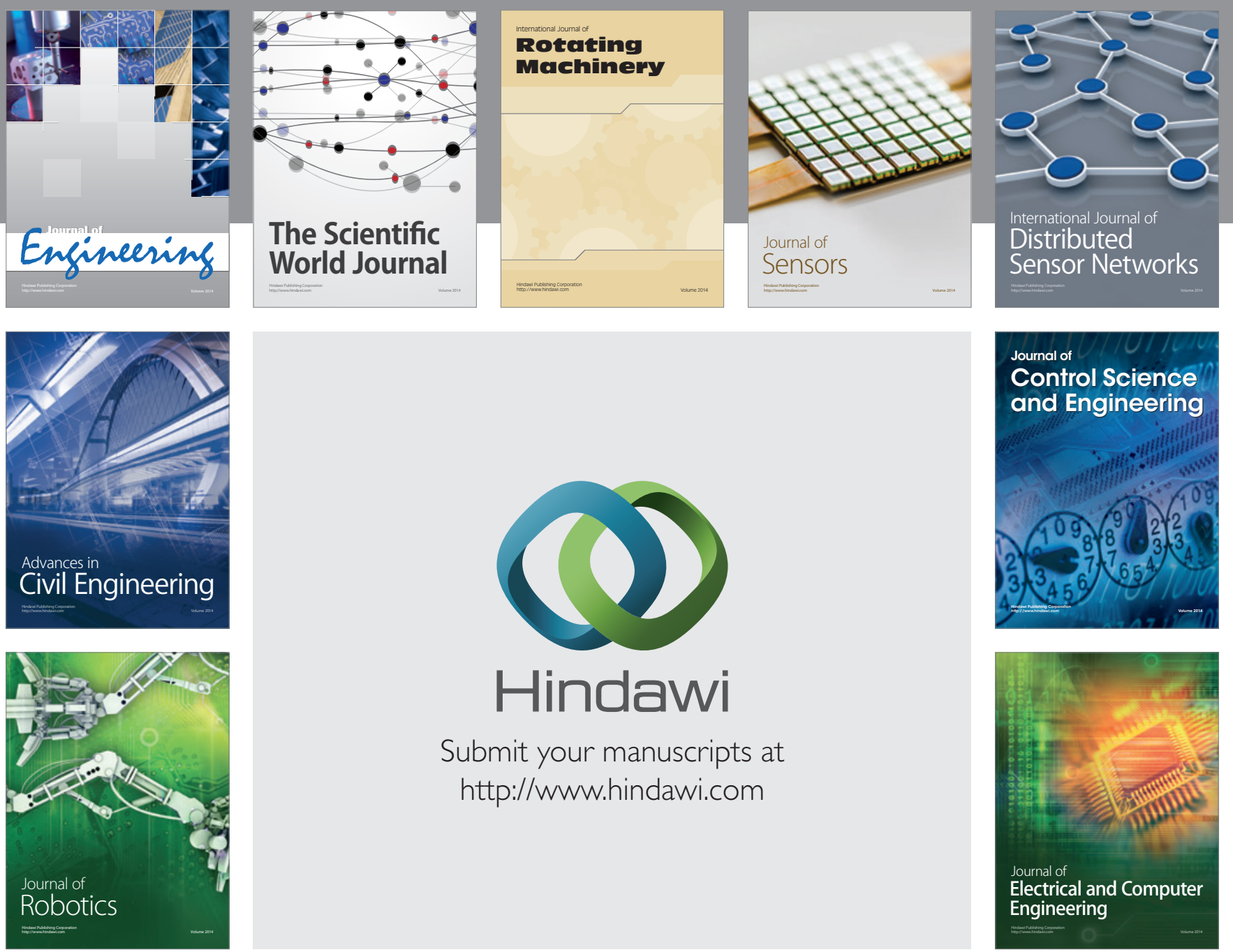

Submit your manuscripts at

http://www.hindawi.com
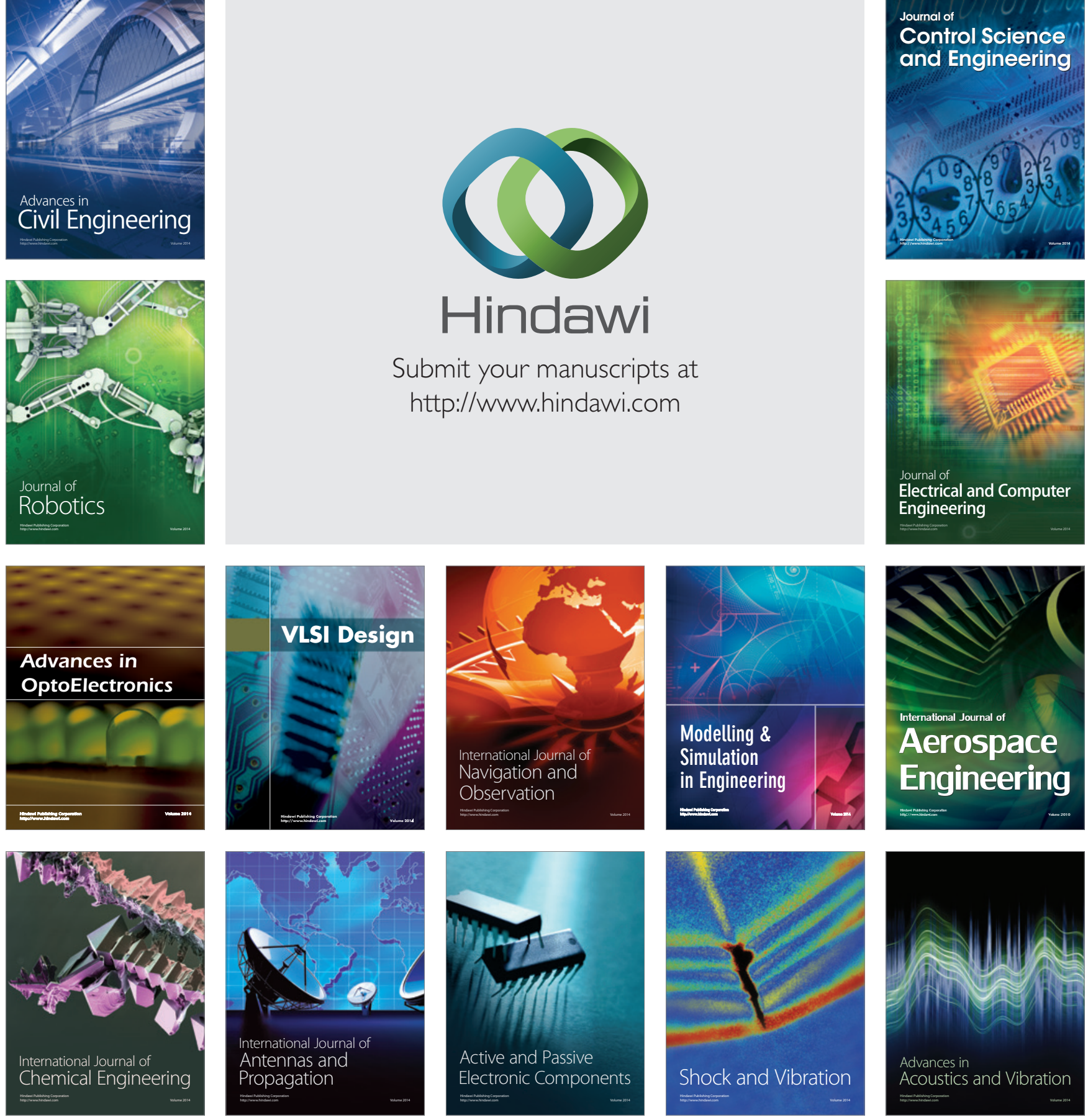